\title{
Diagnóstico del consumo de tabaco en estudiantes de pregrado de la Pontificia Universidad Católica de Valparaíso
}

\author{
LISSETH BARRA C. . , PAOLA FERNÁNDEZ P. ${ }^{\mathrm{b}}$, FELIPE GRANADA G. ${ }^{\mathrm{b}}$, \\ PAULA ÁVILA C. ${ }^{\mathrm{b}}$, JAVIER MALLEA M. ${ }^{\mathrm{b}}$, YENIFFER RODRÍGUEZ M. ${ }^{\mathrm{b}}$
}

\section{Smoking among undergraduate university students}

Background: Smoking is one of the major Public Health problems worldwide. Aim: To study the frequency of tobacco smoking among undergraduate students of a Chilean university. Material and Methods: An opinion survey was sent by e-mail to all undergraduate students of a university, registering gender, age, study years, study area, smoking behavior, motivation (reason for smoking), intention to quit and tobacco law perception. Results: 1,008 (57\% females) out of 11,679 surveys were answered back. Prevalence of active smoking among respondents was $36 \%$, without association with gender, age or years of study. However, students from scientific areas had a lower prevalence. Seventy seven percent of smokers manifested the intention to quit the habit or have started quitting already. Ninety six percent were acquainted with the tobacco law and by $73 \%$ agreed with it. Conclusions: Smoking is highly prevalent among university students. It is necessary to develop strategies for smoking cessation within universities that may prevent or reduce tobacco smoking among students.

(Rev Med Chile 2015; 143: 1343-1350)

Key words: Prevalence; Public Health; Smoking Cessation; Smoking, Students.

E 1 tabaquismo es una enfermedad crónica y adictiva, considerada por la Organización Mundial de la Salud (OMS) como la principal causa de muerte evitable en el mundo ${ }^{1,2}$. Debido a la magnitud de las consecuencias sanitarias que representa, actualmente es un importante problema de salud pública.

Respecto al consumo de tabaco en América, Chile es el país que presenta los valores más altos tanto en la población joven como adulta ${ }^{3}$. A nivel nacional, el año 2012, se realizó la 7a Encuesta Nacional de la Juventud aplicada a jóvenes entre 15 y 29 años, encontrándose una prevalencia de consumo de tabaco de $49,1 \%{ }^{4}$. El consumo de tabaco suele comenzar en la adolescencia, sin embargo, sería durante la etapa adulto joven, para

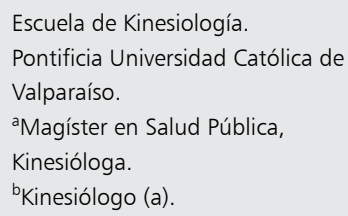

Recibido el 12 de enero de 2015, aceptado el 4 de agosto de 2015

Correspondencia a:

Lisseth Barra Cabello

Escuela de Kinesiología

Pontificia Universidad Católica de Valparaíso

Avda. Universidad 330, Curauma. Teléfonos: 032-2274066

lisseth.barra@pucv.cl muchos jóvenes etapa universitaria, cuando se consolidaría este hábito ${ }^{5,6}$.

A nivel mundial, la prevalencia del consumo de tabaco es variable entre los estudiantes universita$\operatorname{rios}^{7-13}$. En Chile se han realizado dos estudios que consideraron a toda la población universitaria, el primero realizado en la Universidad de Concepción (UdeC) en 1998, que arrojó una prevalencia de $44 \%{ }^{14}$. El segundo, lo realizó la Universidad Austral de Chile (UACh) en el año 2007 revelando una prevalencia de $28,3 \%{ }^{15}$. Al relacionar los años de estudio con el consumo de tabaco, un estudio en la Pontificia Universidad Católica de Chile, mostró que en la carrera de Medicina, a mayor años de estudio, mayor era el consumo de tabaco ${ }^{16}$, datos que se complementan con lo descrito por 
De Grazia et al. en estudiantes de la Universidad de Chile (UCh). Sin embargo, este último estudio describió que en la carrera de Ingeniería de la UCh, el consumo de tabaco disminuía a mayor cantidad de años de estudio ${ }^{17}$.

En relación a las intervenciones que favorecen el cese tabáquico, se debe considerar un tratamiento psicosocial. Para comenzar, resulta relevante conocer la disposición que tienen los fumadores al cese tabáquico y objetivar la dependencia psicosocial del fumador, aspecto que puede ser valorado utilizando "El Test de los Por qué"18, que permite además conocer cuáles son las motivaciones que tienen para continuar con el hábito ${ }^{19}$, ámbito que no ha sido abordado en estudiantes universitarios chilenos.

Considerando la magnitud de este problema, el año 2006 Chile promulgó la Ley de Tabaco respondiendo a las medidas propuestas por el Convenio Marco de la OMS para el Control del Tabaco ${ }^{20,21}$. Luego, el año 2013 se actualiza dicha Ley (Ley N ${ }^{\circ} 20.660$ ) aumentando las restricciones del consumo de tabaco, siendo una de las medidas más controversiales la prohibición de fumar en lugares cerrados de uso público o colectivo. También prohíbe la publicidad del tabaco y de elementos de las marcas relacionadas con dicho producto, extendiéndose a la publicidad indirec$\mathrm{ta}^{3}$. Dado el poco tiempo que llevan en vigencia las modificaciones a la Ley, existen escasos estudios que describan la percepción que tiene la población a dichas modificaciones. Una encuesta realizada por la organización "Chile Libre de Tabaco" indicó que $70,1 \%$ de los encuestados apoya las nuevas restricciones $^{22}$. Sin embargo, la percepción que tiene la población universitaria respecto a la nueva Ley, aún no ha sido descrita.

Desde el 2006 se ha comenzado a potenciar la promoción de la salud en las universidades de Chile, conceptualizada con el término "Universidad Saludable y/o Promotora de la Salud", abarcando cinco temáticas fundamentales, entre ellas; la prevención del consumo de tabaco. En este contexto, se sugiere la realización de un diagnóstico basal para estimar el impacto del consumo de tabaco en la universidad ${ }^{23}$. La Pontificia Universidad Católica de Valparaíso (PUCV), a través de la Dirección de Asuntos Estudiantiles (DAE), cuenta con un programa dedicado al área de calidad de vida, denominado "VIVE SALUD"24, el cual no tiene antecedentes del consumo de tabaco entre sus estudiantes.
De lo expuesto previamente, queda en evidencia que en Chile existen pocos estudios actualizados en relación al consumo de tabaco en estudiantes universitarios. Además, no se han descrito las motivaciones por las cuáles los estudiantes universitarios fuman, la disposición de cambio al cese tabáquico, ni la percepción que tienen respecto a las nuevas medidas de la Ley de Tabaco vigente. Esta información es necesaria para establecer un diagnóstico base que permita medir la efectividad de las intervenciones anti-tabáquicas que se pudiesen implementar en el ambiente universitario y orientar dichas estrategias de la forma más adecuada. Por consiguiente, el objetivo de este estudio es diagnosticar la situación de consumo de tabaco en los estudiantes de pregrado de la PUCV.

\section{Material y Método}

Se realizó un estudio descriptivo utilizando metodología cuantitativa y cualitativa. La población en estudio correspondió a los estudiantes de pregrado de la PUCV matriculados en el segundo semestre del año 2013 (11.770 estudiantes, 54\% sexo masculino).

Para la recolección de datos se utilizó un cuestionario semi-estructurado con instrumentos validados y otras preguntas de interés, entre ellas; sexo, rango etario, carrera y año de ingreso a la universidad. La carrera señalada por los estudiantes se clasificó en una de las tres áreas de estudio definidas (Científica, Humanista y Matemática). Para establecer dicha clasificación previamente se agruparon las facultades de la PUCV en las áreas señaladas de acuerdo al área del conocimiento de mayor afinidad. También, incluyó preguntas relacionadas con la percepción de los estudiantes respecto al grado de conocimiento, acuerdo y opinión en cuanto la nueva ley (ésta última como una pregunta abierta). La conducta fumadora consideró una clasificación basada en el criterio del equipo investigador y las categorías propuestas por la OMS 5 . Las categorías eran: No fumador, Ex-fumador, y Fumador actual, este último subclasificado en fumador diario y ocasional. Para identificar los motivos del hábito tabáquico y la etapa de cambio en que se encontraba el estudiante frente a este hábito, se utilizó el "Test de los Por qué" y "Prochaska y Di Clemente", respectivamente. 
El cuestionario fue enviado por la DAE a 11.679 estudiantes a través de sus correos electrónicos personales. Para obtener una muestra de tamaño adecuado, como mínimo 977 estudiantes debían contestarla. Este resultado fue obtenido al utilizar la fórmula de cálculo de tamaño de muestra de estudios de prevalencia considerando un nivel de confianza de $95,5 \%$ y un error muestral de $3 \%{ }^{25}$.

\section{Aspectos éticos}

Previo al envío de la encuesta, todos los estudiantes de pregrado recibieron un correo electrónico con los objetivos de la investigación y destacando que la participación era de carácter voluntaria y que no pretendía juzgar el comportamiento individual. La encuesta fue anónima, y en el caso de quienes quisieran recibir sus resultados, podían obtenerlos enviando su nombre y un correo electrónico de contacto. A fin de resguardar la confidencialidad, los datos personales solicitados no fueron publicados ni expuestos de ninguna forma. La información obtenida fue de carácter confidencial y sólo analizada por el equipo investigador.

\section{Análisis de datos}

Los datos fueron almacenados y analizados a través de los programas Google Drive ${ }^{\circledR}$ y Microsoft Excel $2010^{\circledR}$. Para el análisis estadístico de datos de variables medidas en forma cuantitativa, se utilizó el programa estadístico Stata/SE $12.0^{\circledR}$. Los resultados fueron analizados con estadística descriptiva. Además, se realizó análisis comparativo usando la distribución $\chi^{2}$, con un nivel predefinido de significación para las pruebas estadísticas de $\mathrm{p}<0,05$. Para el análisis de la variable medida en forma cualitativa (opiniones de los estudiantes), se realizó un análisis de contenido.

\section{Resultados}

\section{Descripción de la muestra}

Un total de 1.008 estudiantes contestaron la encuesta. De éstos 56,9\% fueron del sexo femenino. La mayoría de los estudiantes tenía entre 22 y 24 años (41\%), llevaba entre 3 y menos de 6 años estudiando en la institución (40,7\%) y $37,4 \%$ pertenecía al área matemática.

\section{Consumo de tabaco}

La prevalencia de fumadores actuales de los estudiantes de la PUCV fue de $35,7 \%$, cifra que representa tanto a fumadores diarios $(17,1 \%)$ como a fumadores ocasionales (18,6\%) (Figura 1).

El consumo de tabaco de las variables sexo, rango etario y años de estudio de los estudiantes, no presentaron diferencias estadísticamente significativas $(p>0,05)$. Sin embargo, según área de estudio, el área Científica, con una prevalencia de consumo de tabaco de $26,5 \%$, fue significativamente menor en comparación con las otras áreas $(\mathrm{p}=0,001)($ Tabla 1$)$.

\section{Motivaciones para el consumo de tabaco}

El "Test de los Por qué" reveló que la principal motivación para fumar tanto de mujeres como de hombres fue ser Fumador social con $71,6 \%$, seguido de Manejo de Tensión con 22,8\% (Figura 2).

\section{Etapas de cambio del hábito tabáquico según Prochaska y Di Clemente}

De los fumadores actuales, $33,7 \%$ de los estudiantes se hallaba en etapa de Pre-contemplación, 43,7\% en Contemplación y 22,6\% en Preparación. Considerando a los estudiantes que declararon ser Ex-fumadores, $22 \%$ se encontraba en etapa

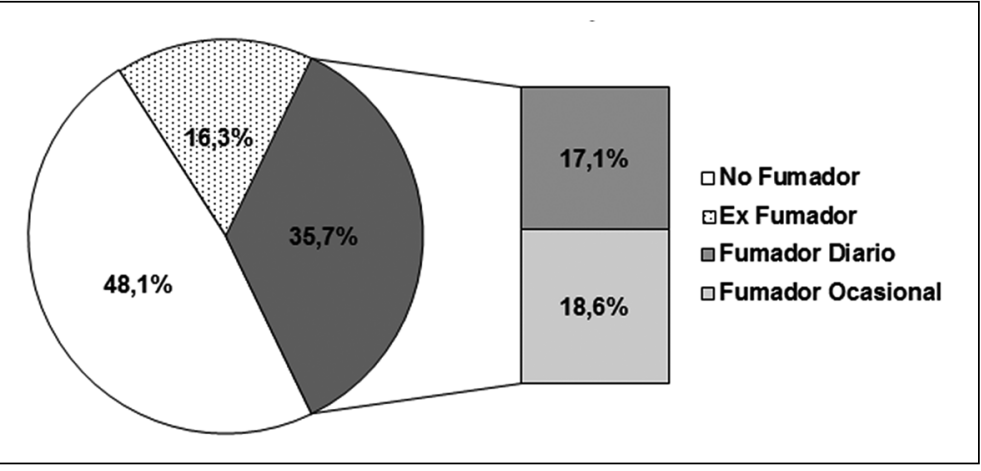

Figura 1. Conducta Fumadora de los Estudiantes de Pregrado de la PUCV. 
Tabla 1. Consumo de tabaco según sexo, rango etario, años y área de estudio

\begin{tabular}{|c|c|c|c|c|c|}
\hline Variable & & $n_{1}$ & $n_{2}$ & $\%$ & p value \\
\hline \multirow[t]{2}{*}{ Sexo } & Femenino & 574 & 204 & $35,54 \%$ & \multirow[t]{2}{*}{$p=0,954$} \\
\hline & Masculino & 434 & 155 & $35,71 \%$ & \\
\hline \multirow[t]{4}{*}{ Edad } & Menos de 19 años & 67 & 25 & $37,31 \%$ & \multirow[t]{4}{*}{$p=0,969$} \\
\hline & Entre 19 y 21 años & 374 & 130 & $34,76 \%$ & \\
\hline & Entre 22 y 24 años & 413 & 148 & $35,84 \%$ & \\
\hline & 25 o más años & 154 & 56 & $36,36 \%$ & \\
\hline \multirow[t]{4}{*}{ Años de estudio } & < a 1 año & 184 & 68 & $36,96 \%$ & \multirow[t]{4}{*}{$p=0,517$} \\
\hline & Entre 1 y $<3$ años & 305 & 108 & $35,54 \%$ & \\
\hline & Entre 3 y $<6$ años & 410 & 151 & $36,68 \%$ & \\
\hline & $\geq$ a 6 años & 109 & 32 & $29,35 \%$ & \\
\hline \multirow[t]{3}{*}{ Área de estudio** } & Área Humanista & 307 & 126 & $41,04 \%$ & \multirow[t]{3}{*}{$p=0,0001 *$} \\
\hline & Área Científica & 324 & 86 & $26,54 \%$ & \\
\hline & Área Matemática & 377 & 147 & $38,99 \%$ & \\
\hline
\end{tabular}

$\mathrm{n}_{1}$ : Cantidad de encuestas respondidas/ $\mathrm{n}_{2}$ : Cantidad de Fumadores / \%: Prevalencia consumo de tabaco expresada en porcentaje. *Estadísticamente significativo. **En esta clasificación las facultades de la PUCV se agruparon de la siguiente forma: Área Científica: Facultad de Ciencias, Facultad de Ciencias del Mar y Geográficas, Facultad de Ciencias Agronómicas y de los Alimentos. Área Humanista: Facultad Eclesiástica de Teología, Facultad de Filosofía y Educación, Facultad de Derecho. Área Matemática: Facultad de Ingeniería, Facultad de Arquitectura y Urbanismo, Facultad de Ciencias Económicas y Administrativas.

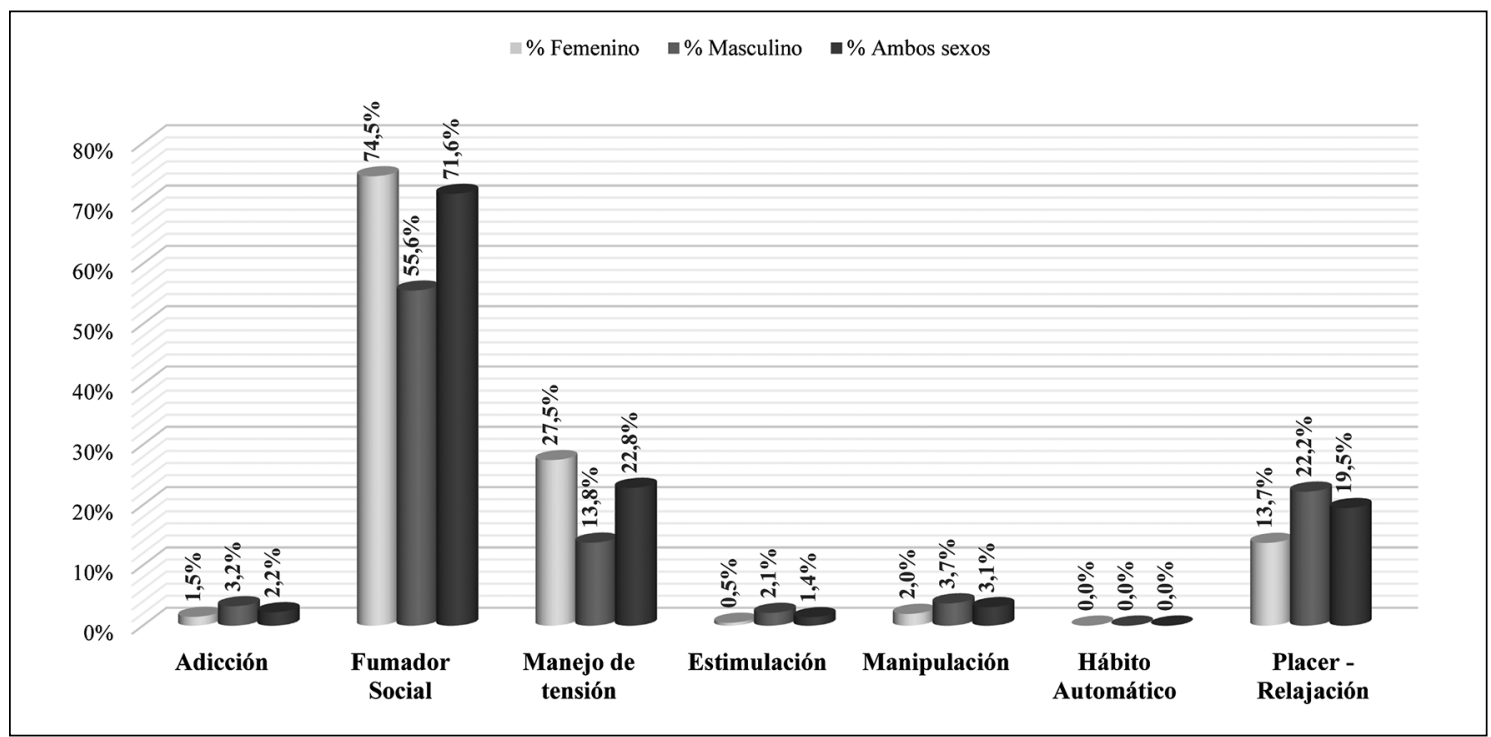

Figura 2. Motivaciones por las cuáles los Estudiantes de Pregrado de la PUCV fuman, según resultados obtenidos en el "Test de los por qué". Distribución según Sexo. El "Test de los Por qué" permite evaluar el rol que juega el consumo de tabaco en cada fumador y conocer cuáles son las motivaciones que tiene cada uno para continuar con el hábito; presenta 7 posibles categorías, no excluyentes entre ellas, es decir un fumador podría presentar más de una motivación para fumar

de Acción y 78\% en Mantención. Considerando ambos grupos (Fumadores y Ex-fumadores) un $76,9 \%$ declaró tener la intención o haber comenzado a abandonar el hábito tabáquico.

\section{Ley de tabaco}

En relación al conocimiento que tuvieron los estudiantes respecto a la actual Ley de Tabaco, $95,5 \%$ declaró conocerla, de ellos, $51,1 \%$ en su 
Tabla 2. Opiniones de estudiantes universitarios respecto a la actual Ley de Tabaco

\begin{tabular}{|c|c|}
\hline \multicolumn{2}{|l|}{ Fumadores } \\
\hline $\begin{array}{l}\text { De acuerdo con la Ley } \\
\text { de Tabaco }\end{array}$ & $\begin{array}{l}\text { "Permite reducir el consumo de tabaco, puesto que ahora cuando salgo, ya no fumo" } \\
\text { "Permite que el fumador, y especialmente, el no fumador tengan más espacios libres de humo } \\
\text { de tabaco, lo cual ayuda a disminuir las consecuencias sanitarias que éste trae consigo, tanto a } \\
\text { la persona, como al medio ambiente" }\end{array}$ \\
\hline $\begin{array}{l}\text { Parcialmente de acuerdo } \\
\text { con la Ley de Tabaco }\end{array}$ & $\begin{array}{l}\text { "La nueva Ley de Tabaco protege los derechos de los no fumadores, pero transgrede el dere- } \\
\text { cho de los fumadores, ya que éstos no pueden consumir cigarrillos en una gran cantidad de } \\
\text { espacios" } \\
\text { "Hay lugares en los que no se debiese prohibir el consumo de tabaco, tales como bares, pubs, } \\
\text { discoteques o en su defecto, debiese existir espacios para fumadores y no fumadores en un } \\
\text { mismo local" }\end{array}$ \\
\hline $\begin{array}{l}\text { Desacuerdo con la Ley } \\
\text { de Tabaco }\end{array}$ & $\begin{array}{l}\text { "Atenta contra la libertad de decisión de los fumadores, ya que no deja ningún espacio público } \\
\text { (cerrado) apto para el consumo" }\end{array}$ \\
\hline \multicolumn{2}{|l|}{ No fumadores } \\
\hline $\begin{array}{l}\text { De acuerdo con la Ley } \\
\text { de Tabaco }\end{array}$ & $\begin{array}{l}\text { "Aumenta los espacios públicos (y cerrados) libres de humo de tabaco, lo cual permite que } \\
\text { personas puedan estar en lugares en los antes les era imposible estar por la calidad del aire" } \\
\text { "Generaba un gran desagrado asistir a lugares de diversión por el olor que ahí había, ahora ya } \\
\text { no sucede esto" } \\
\text { "Permite mayor educación respecto a los daños del tabaco. Así como situar al fumador en una } \\
\text { posición más consciente y empática con relación al cigarro y la salud de todos, abriendo las } \\
\text { posibilidades de un futuro país más consciente y saludable" }\end{array}$ \\
\hline $\begin{array}{l}\text { Parcialmente de acuerdo } \\
\text { con la Ley de Tabaco }\end{array}$ & $\begin{array}{l}\text { "No sólo en los lugares públicos cerrados debería prohibirse fumar, sino que además en los } \\
\text { abiertos, ya que es muy dañino cuando las personas por ejemplo fuman en la calle" }\end{array}$ \\
\hline $\begin{array}{l}\text { Desacuerdo con la Ley } \\
\text { de Tabaco }\end{array}$ & "Se restringe la libertad de decisión" \\
\hline
\end{tabular}

totalidad y $44,4 \%$ parcialmente. Al solicitar de manera voluntaria la opinión que tenían respecto a la actual Ley de Tabaco, 74,5\% de los estudiantes respondió la pregunta. Al realizar el análisis de contenido sólo se observaron diferencias en el discurso que refirieron al agrupar estas opiniones de acuerdo al grado de acuerdo con la Ley.

Entre los estudiantes fumadores, 58,2\% refirió estar de acuerdo con la actual Ley. Sus fundamentos fueron que permite moderar y en muchos casos, reducir el consumo de tabaco. Declararon que les gusta la idea de proteger al medio ambiente, permitiendo que todas las personas pudieran respirar en un ambiente menos contaminado. El 29,5\% de los fumadores estuvo parcialmente de acuerdo con la Ley. Éstos la consideraron un poco exagerada, pues reduce excesivamente los espacios para fumadores $y$, pese a que disminuye el consumo de tabaco, debieran existir ambientes separados para fumadores y no fumadores. En cambio, $11,4 \%$ de los fumadores no estuvo de acuerdo con la Ley, señalando que se restringe excesivamente la libertad y que a su vez, transgrede los derechos de los fumadores (Tabla 2).

El $82,4 \%$ de los estudiantes no fumadores declararon estar de acuerdo con la Ley. Éstos argumentaron que las medidas aumentan los espacios libres de humo de tabaco, permitiendo así asistir a muchos lugares donde previamente no lo hacían (pubs, discoteques, bar-restaurant, etc.) por entre otras razones, el desagrado que les producía el humo y el daño que éste les generaba. Junto con esto, destacaron la labor educativa que genera la Ley, creando una mayor empatía por parte del fumador hacia el que no lo es, haciendo énfasis en los niños. El 11,9\% refirió estar parcialmente de acuerdo, justificando su opinión en que ésta debiese ser más estricta, haciendo alusión a que debería limitar espacios públicos abiertos, por ejemplo el fumar en la calle, ya que atenta contra la salud de las personas que no fuman pero reciben el humo. El 2,8\% declaró estar en desacuerdo con la Ley, argumentando que se restringe la libertad de decisión (Tabla 2). 


\section{Discusión}

La prevalencia actual de fumadores en estudiantes de pregrado de la PUCV fue de $35,7 \%$, cifra que comparada con estudios similares de otras casas de estudio, fue mayor a la UACh $(28,3 \%)^{15}$, pero menor a la UdeC $(44 \%)^{14}$. En Chile el nivel educacional alto (12 o más años de estudios) es el que presenta la mayor prevalencia de consumo de tabaco ${ }^{26}$, siendo la época universitaria una etapa clave para la prevención.

Al comparar prevalencia según sexo, el presente estudio demostró que no existe diferencia significativa entre el consumo de tabaco de hombres y mujeres, situación que coincide con lo hallado en otros estudios universitarios ${ }^{6,16}$. Confirmando así la actual tendencia en Chile de una disminución en la brecha del consumo de tabaco entre hombres y mujeres ${ }^{26,27}$, la cual es aún menor en el nivel educacional alto, que corresponde a la población en estudio.

En relación a la edad no se encontró asociación con el consumo de tabaco, lo que difiere de lo hallado en otros estudios en universita$\operatorname{rios}^{6,28}$, en los cuales a mayor edad, mayor era el consumo de tabaco. Tampoco se observó una relación entre años de estudio y consumo de tabaco, situación que concuerda con el estudio de Zuzulich et $\mathrm{al}^{28}$.

Sin embargo, el consumo de tabaco sí está asociado al área de estudio al que pertenezca el alumno, resultados que concuerdan con los estudios de Morales et al. y Sotomayor et al. Esta situación debiese ser considerada para orientar las campañas antitabaco que se pudiesen desarrollar a nivel universitario.

En relación a las motivaciones que presentaron los fumadores, el "Test de los Por qué" reveló información de la cual no se encontró precedente en estudios de otras universidades tanto a nivel nacional como internacional. El hecho que tanto para mujeres como hombres la principal motivación sea el aspecto social, facilita que las campañas masivas sean consideradas una buena herramienta de prevención, ya que permitiría abarcar a la mayor cantidad de fumadores.

Respecto a las Etapas de Cambio según Prochaska y Di Clemente, esta investigación reveló que existe una gran cantidad de estudiantes que tienen la intención de abandonar el hábito tabáquico, justificando de esta forma, la importancia de apoyarlos en su decisión mediante intervenciones antitabáquicas.

En cuanto a la actual ley de tabaco, un alto porcentaje de estudiantes declaró conocerla y estar de acuerdo con ella. Lo que podría explicar que la mayoría de las personas reconocen que el consumo de tabaco es un peligro para la salud, tanto de quienes fuman como de quienes están expuestos al humo. Incluso, la mayoría de los fumadores consideran que la ley ha sido un beneficio, puesto que los ayuda a disminuir su consumo. Sería interesante que futuros estudios pudiesen evaluar el impacto de estas políticas en la población universitaria.

La limitación de este estudio se relaciona con la aplicación de una encuesta online, dado que las respuestas pudieron provenir sólo de estudiantes interesados en el tema del tabaco. En la muestra obtenida las mujeres fueron quienes más respondieron la encuesta, pese a que en el universo la mayoría eran varones. Aunque se podría haber utilizado una muestra estratificada según variables de interés aplicada de manera presencial, se decidió utilizar una encuesta enviada de manera virtual en consideración a las siguientes ventajas: posibilidad de abarcar a toda la población, optimización de los recursos, rapidez en la obtención de los resultados, disminución del sesgo del entrevistador y facilitar el acceso a la encuesta a través de uno de los medio de comunicación más utilizado actualmente por los jóvenes universitarios. A fin de favorecer la participación de todos los estudiantes de pregrado, se promocionó la investigación previa al envío de la encuesta.

Esta investigación cumple con el objetivo propuesto, aportando los primeros antecedentes respecto de la problemática del tabaco entre los estudiantes de pregrado de la PUCV. La información podría ser utilizada para orientar futuras intervenciones antitabáquicas en el marco del programa de promoción de la salud ya existente en esta casa de estudios.

\section{Referencias}

1. Organización Mundial de la Salud. Informe OMS sobre la Epidemia Mundial del Tabaquismo. [Online].; 2013 [citado el 3 de mayo de 2013]. Disponible en: http:// www.who.int/tobacco/global_report/2013/summary/ es/ 
2. Ministerio de Salud. Encuesta Mundial de tabaquismo en jóvenes. Ministerio de Salud de Chile, Departamento de Epidemiología; 2005.

3. Ministerio de Salud. Manual para la fiscalización de la Ley de Tabaco $\mathrm{N}^{\circ} 19.419$ Modificada por la Ley $\mathrm{N}^{\circ}$ 20.660. Oficina de Tabaco división de políticas públicas y división; 2013.

4. INJUV. 7ma Encuesta Nacional de la Juventud. [Online].; 2012 [citado el 22 de julio de 2013]. Disponible en: http://www.injuv.gob.cl/portal/wp-content/files_mf/ septimaencuestanacionaljuventud2.pdf

5. Ministerio de Salud. Tabaquismo en jóvenes chilenos: Tristes récords. [Online].; 2012 [citado el 18 de mayo de 2013]. Disponible en: http://www.chilelibredetabaco. cl/wp-content/uploads/2012/10/Tabaquismo-en-Jovenes-Chilenos.pdf

6. Morales G, Valle C, Orellana Y, Soto A, Ivanovic D. "Prevalencia del Consumo de drogas en estudiantes universitarios que cursan primer y cuarto año". Rev Chil Salud Pública 2011; 139: 1573-80.

7. Ohmi H, Okizaki T, Meadows M, Tereyama K, Mochizuki Y. An exploratory analysis of the impact of a university campus smoking ban on staff and student smoking habits in Japan. Tobacco Induced Diseases. 2013; 11 (19).

8. Chkhaidze I, Maglakelidze N, Maglakelidze T, Khaltaev N. Prevalence of and factors influencing smoking among medical and non-medical students in Tbilisi, Georgia. J Bras Pneumol 2013; 39(5): 579-84.

9. Ledo-Varela M, de Luis Román D, González-Sagredo M, Izaola O, Conde R, Aller de la Fuente R. Características nutricionales y estilo de vida en universitarios. Nutr Hosp 2011; 26 (4): 814-8.

10. Morales Z, Pascual L, Garrido R. Valoración de sesgos atencionales visuales en una muestra de fumadores universitarios. Adicciones 2013; 25(2): 163-9.

11. Noonan D. A descriptive study of waterpipe smoking among college students. Journal of the American Association of Nurse Practitioners 2013; 25 (1): 11-5.

12. Sutfin E, McCoy T, Berg C, Champion H, Helme D, O’Brien M, et al. Tobacco use by college students: a comparison of daily and nondaily smokers. Am J Health Behay 2012; 36 (2): 218-29.

13. Fabelo J, Iglesias S, Cabrera R, Maldonado M. Tobacco and Alcohol Consumption among Health Sciences Students in Cuba and Mexico. MEDICC Review 2013; 15(4): 18-23.

14. Sotomayor H, Behn V, Cruz M, Naveas R, Sotomayor C, Fernández $\mathrm{P}$, et al. Tabaquismo en académicos, no académicos y estudiantes de la Universidad de Concepción. Rev Med Chile 2000; 128(9): 977-84.
15. Martínez M, Leiva A, Sotomayor C, Victoriano T, Von Chrismar A, Pineda S. Factores de riesgo cardiovascular en estudiantes de la Universidad Austral de Chile. Rev Med Chile 2012; 140 (2): 426-35.

16. Romero M, Santander J, Hitschfeld M, Labbe M, Zamora V. Consumo de Tabaco y Alcohol entre los estudiantes de medicina de la Pontificia Universidad Católica de Chile. Rev Med Chile 2009; 137: 361-8.

17. De Grazia J, Faivovich D, Alarcón F, Díaz R, Yentzen G, Kunstmann S. Prevalencia de tabaquismo y actitud de cambio frente al hábito tabáquico en universitarios chilenos: Importancia de la formación médica. Rev Med Chile 2009; 13 (2): 72-81.

18. Bello S, Flores A, Bello M, Chamorro H. Diagnóstico y tratamiento psicosocial del tabaquismo. Rev Chil Enferm Respir 2009; 25 (4): 218-30.

19. Ministerio de Salud. La cesación del consumo de tabaco [Online]; 2003 [citado el 18 de mayo de 2013]. Disponible en: http://www.minsal.gob.cl/portal/url/ item/75fd0bb79ec68b90e04001011f01201c.pdf.

20. Organización Mundial de la Salud. Convenio Marco de la OMS para el control del tabaco [Online]; 2003 [citado el 2 de febrero de 2014]. Disponible en: http://whqlibdoc.who.int/publications/2003/9243591010.pdf?ua=1

21. Organización Panamericana de la Salud. Informe sobre el control del tabaco para la Región de las Americas. [Online]; 2013 [citado el 2 de febrero de 2014]. Disponible en: http://www.paho.org/hq/index.php?option=com_docman\&task=doc_download\&gid=23415\&Itemid=270\&lang=en.

22. Amarales L. Estudio de Opinión Pública sobre la nueva Ley de tabaco [Online]; 2013 [citado el 13 de septiembre de 2013]. Disponible en: http://www.chilelibredetabaco. cl/wp-content/uploads/2013/05/encuesta-flash-tabaco-demoscopica-abril-2013.pdf.

23. Lange I, Vio F. Guía para universidades saludables y otras instituciones de Educación Superior. [Online]; 2006 [citado el 5 de mayo de 2013]. Disponible en: http://www.eligevivirsano.cl/wp-content/ uploads/2012/01/Guia-Universidades-Saludables_INTAOPS.pdf

24. Comunicaciones DAE. Vive Salud. [Online]; 2013 [citado el 4 de septiembre de 2013]. Disponible en: http:// vra.ucv.cl/dae/?page_id=1391.

25. Aguilar S. Fórmulas para el cálculo de la muestra en investigaciones de salud. Salud en Tabasco Secretaria de Salud del Estado de Tabasco 2005; 11 (1-2): 333-8.

26. Ministerio de Salud. Segunda Encuesta Nacional de Salud. [Online]; 2009-2010 [citado el 7 de marzo de 2013]. Disponible en: http://www.redsalud.gov.cl/portal/url/ item/99bbf09a908d3eb8e04001011f014b49.pdf. 
27. Departamento de Salud Pública PUC. Resultados de la primera Encuesta de Salud. [Online]; 2003 [citado el 4 de marzo de 2014]. Disponible en: http://epi.minsal.cl/ epi/html/invest/ens/informefinalens.pdf.
28. Zuzulich M, Cabieses B, Pedrals N, Contreras L, Martínez D, Muñoz M. Factores asociados a consumo de tabaco durante el último año en estudiantes de educación superior. Invest Educ Enferm 2010; 28 (2): 232-9. 